\section{Carcinoma Arising in a Lipomatous Pseudohypertrophic Pancreas}

Brit. med. F., 1968, 3, 293

Lipomatous pseudohypertrophy of the pancreas is such a rare lesion that it is not referred to in many recent textbooks of pathology. The association of this condition with a pancreatic carcinoma is even rarer, and hence recording of a single case does appear to be justified.

\section{CASE REPORT}

A woman aged 71 was admitted to hospital with tachycardia, gangrene of the toes of the right foot, and a palpable mass below the right costal margin. Malignancy was suspected but a primary was not identified. Terminally she developed uraemia, leucocytosis, and pyrexia, and she died one month after admission in cardiac failure.

Necropsy.-There were multiple recent pulmonary emboli, pulmonary congestion, and oedema. Several toes of the right foot showed early gangrene. The thyroid was enlarged and adenomatous, weighing $116 \mathrm{~g}$. The pancreatic duct did not admit a probe. The pancreas was greatly enlarged and appeared to consist almost exclusively of fat tissue. It measured 23 by 5 by $4 \mathrm{~cm}$. and weighed 250 g. (normal 65-135 g.). In its centre there was a smooth-walled cyst, measuring 4.5 by $3.5 \mathrm{~cm}$. Caudally the cyst wall was adjoined by a greyish, moderately soft neoplasm, its crescent-shaped cut surface measuring 2 by $1 \mathrm{~cm}$. (Fig. 1). Large necrotic secondary deposits were present in the liver, the largest of which, measuring $10 \mathrm{~cm}$. in diameter, projecting from the anterior margin of the right lobe (this metastasis had been palpable during

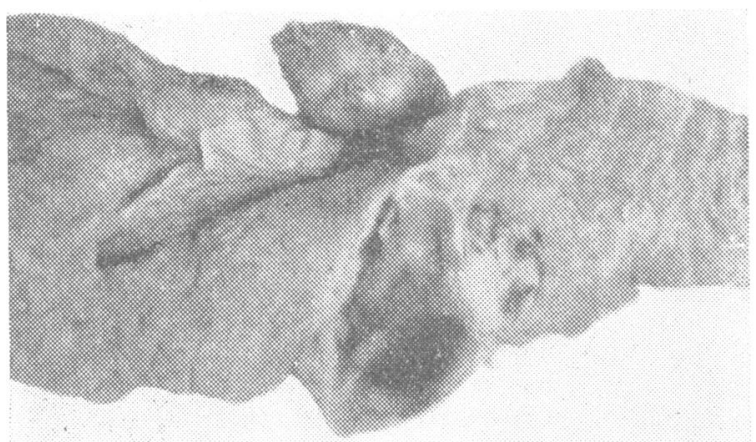

FIG. -1.-Central area of pancreas showing fatty parenchyma, retention cyst bordered distally by carcinoma, and neoplastic parapancieatic lymph node. Slightly reduced.

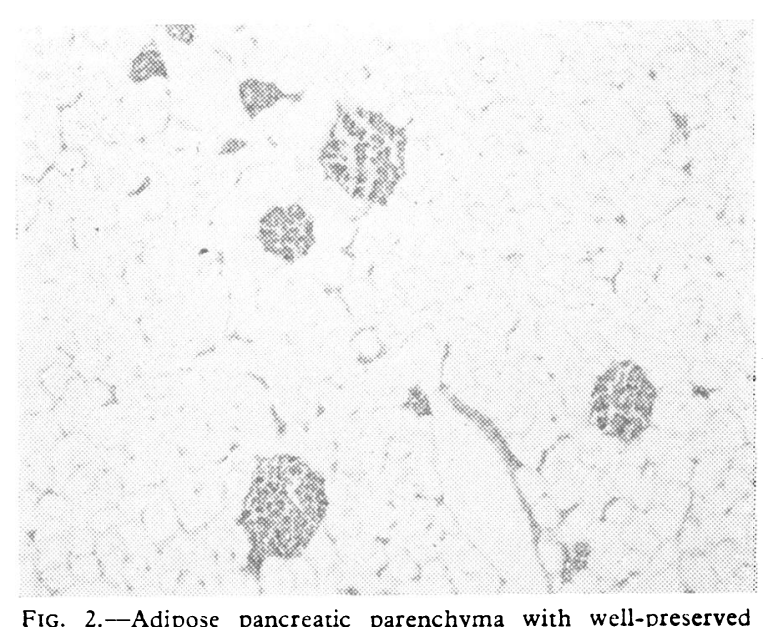

FIG. 2.-Adipose pancreatic parenchyma with well-preserved islets of Langerhans. (Haematoxylin-eosin. $\times 32$.) life). Neoplastic lymph nodes were present along the pancreas and upper abdominal aorta, and in the porta hepatis. The spleen showed multiple small subcapsular infarctions. Otherwise the viscera were within normal limits, and examination of skull and vertebral column revealed no abnormalities.

Microscopical Examination Report.-The lining of the pancreatic cyst has largely desquamated, but where present it consists of a single row of columnar cells. The pancreatic tumour is a poorly differentiated scirrhous adenocarcinoma. It is situated outside the cyst wall, and the secondary deposits in the liver and abdominal lymph nodes show similar tumour tissue. The pancreatic ducts are of average calibre or slightly distended, and are patent. In many areas the pancreatic tissue consists exclusively of fat with scattered well-preserved islets of Langerhans (Fig. 2). In other areas there are small remaining groups of exocrine glands, frequently arranged around pancreatic ducts. Apart from congestion the liver shows nothing of note.

\section{Discussion}

The scanty literature of lipomatous pseudohypertrophy of the pancreas has been surveyed in a previous paper (Salm, 1960). Probably no more than four acceptable cases are on record. Clinically, fibrocystic and coeliac disease have to be excluded in each instance showing steatorrhoea.

The exocrine pancreatic parenchyma is very prone to undergo fat replacement, which can be observed, for example, in diabetes and in old age. In all such cases, however, the organ is either of normal size or smaller than normal.

In lipomatous pseudohypertrophy the pancreas is very considerably increased in size and weight, as in the present case, in which the pancreatic weight was increased to about four times the weight of the organ in old age-that is, in lipomatous pseudohypertrophy there is no mere fat replacement of the parenchyma, but an almost complete atrophy of the exocrine tissues is associated with a very considerable excess of adipose tissue. Its causation is unknown. The endocrine tissues do not participate in the glandular atrophy, and the islets of Langerhans survive among the adipose tissues in normal numbers. It is evident that in the case described sufficient exocrine tissue survived to prevent the occurrence of steatorrhoea.

In the previously observed case almost the entire ductal system of the pancreas had been filled with an arborescent calcareous cast, and a scirrhous adenocarcinoma had arisen in the pancreatic head and had disseminated widely (Salm, 1960). In the present case a simple retention cyst was found in the centre of the organ, and a scirrhous adenocarcinoma had developed distal and adjacent to it.

The association of calculi and malignancy is a well-known one, as is exemplified in the gall bladder, urinary bladder, and renal pelvis. In the present case calcareous concretions were absent, but it is noteworthy that the tumour developed immediately distal to the cyst wall, where obstruction to the flow of pancreatic secretion can be assumed to have been maximal.

Thromboembolic lesions, as found in spleen, lungs, and foot, are often associated with carcinoma of the pancreas.

I am indebted to Dr. J. D. Hardy for the clinical details. My thanks are due to Professor R. A. Willis for confirming the diagnosis and to Mr. W. M. Seymour for his technical and photographic work.

R. SALM, M.D., F.C.PATH., Consultant Histopathologist, Royal Cornwall Hospital (Treliske), Truro, Cornwall. 